\title{
AGGREGATE MODELING FOR FLOW TIME PREDICTION OF AN END-OF-AISLE ORDER PICKING WORKSTATION WITH OVERTAKING
}

\author{
Ricky Andriansyah \\ Pascal Etman \\ Jacobus Rooda \\ Systems Engineering Group \\ Eindhoven University of Technology \\ Den Dolech 2, Whoog 0.127, Eindhoven, 5600 MB, THE NETHERLANDS
}

\begin{abstract}
An aggregate modeling methodology is proposed to predict flow time distributions of an end-of-aisle order picking workstation in parts-to-picker automated warehouses with overtaking. The proposed aggregate model uses as input an aggregated process time referred to as the effective process time in combination with overtaking distributions and decision probabilities, which we measure directly from product arrival and departure data. Experimental results show that the predicted flow time distributions are accurate, with prediction errors of the flow time mean and squared coefficient of variation less than $4 \%$ and $9 \%$, respectively. As a case study, we use data collected from a real, operating warehouse and show that the predicted flow time distributions resemble the flow time distributions measured from the data.
\end{abstract}

\section{INTRODUCTION}

An automated warehouse is a network of processing units that store, transport, and consolidate vast amount of products. Some typical processing units in such a warehouse are automated storage/retrieval systems, automated transport systems (e.g., conveyors, automated guided vehicles), and order picking workstations. For such a system, performance evaluation is essential to provide feedback about how a specific design or operational policy performs compared with the requirements, and how it can be improved (Gu, Goetschalckx, and McGinnis 2010).

This paper focuses on performance analysis of one particular processing unit of a parts-to-picker automated warehouse, namely the order picking workstation. An order picking workstation is a crucial value-adding processing unit that collects numerous products retrieved from the storage area to fulfill customer orders. We are mainly interested in predicting the mean and variability of product and order flow times at such a workstation. Flow time distribution gives an important insight on warehouse reliability in meeting customer due dates.

Some previous works on flow time prediction of different types of order picking workstations are available. Koo (2009) compared the mean order flow time and the mean order pick rate of a zone picking system, a bucket brigade picking, and a combination of the two called zoned bucket brigade picking. Using a number of simulation experiments, he showed that under certain assumptions the zoned bucket brigade performs better than the other two systems. Yu and De Koster $(2008,2009)$ developed an approximation method based on a $G / G / m$ queueing network to evaluate the mean order flow time in a pick-and-pass order picking system. Their method produces accurate prediction of mean order flow time, which is practical for quick evaluation of alternative system designs.

There is only limited literature on performance analysis that considers both the mean and the variability of flow times for an order picking workstation. A performance analysis method that is able to quantify these two measures will provide a better insight on the performance of the workstation. In our previous work (Andriansyah, Etman, and Rooda 2009), we proposed a method based on aggregate process time for predicting the performance of an end-of-aisle, unit-load order picking workstation. Assuming a FIFO (First-In-First-Out) processing at the workstation, we were able to predict the mean 
and the variability of product and order flow times. However, overtaking of products and orders, which often occurs in practice, were not taken into account.

In this paper we extend our previous work on performance analysis of an end-of-aisle order picking workstation. We relax the assumption of FIFO processing, allowing products and orders to overtake at the workstation. We propose a simplified simulation model that only requires limited data obtainable from the shop-floor. We refer to this model as the aggregate model. The key aspect of this model is that we do not model in detail the stochastic behavior that are typically difficult to quantify (Rouwenhorst et al. 2000), e.g., the picking time, picking faults, setup times, equipment failures, etc. Instead, these are all aggregated into a so-called EPT (Effective Process Time) (Hopp and Spearman 2008). We measure the EPTs directly from arrival and departure times of products using a sample path equation. Overtaking distributions and a so-called decision probability are also measured. The aggregate model uses the EPT distribution, the overtaking distributions, and the decision probability as input. We show that the aggregate model predicts the mean and variability of product and order flow time with satisfactory accuracy.

The remainder of this paper is organized as follows. Section 2 describes the end-of-aisle order picking workstation. Section 3 describes the proposed aggregate model. Section 4 elaborates a number of simulation experiments to validate the aggregate model. Section 5 presents a case study in which the proposed aggregate modeling methodology is applied to data from a real, operating order picking workstation. Finally, Section 6 concludes the paper.

\section{END-OF-AISLE ORDER PICKING WORKSTATION}

A typical layout of a goods-to-men automated warehouse is shown in Figure 1(a). We consider an end-of-aisle, unit-load order picking workstation as shown in Figure 1(b), which is a part of the automated warehouse. Products are delivered in totes via conveyors to the workstation. A product tote contains items of the same SKU (Stock Keeping Unit). An order consists of a number of SKUs to be picked, which is referred to as the order length. When a picker is idle and the required product tote is present, the tote is sent to the picker. The picker picks the number of required items out of the product tote and puts them in an order tote. Note that the picker processes one order at a time. The order being processed is called the active order, and the totes belonging to this order are referred to as the active totes. If there are no active totes present in the buffer, then the picker will be idle even though totes for other orders may be available. Product totes that still contain items after picking are returned to the storage area.

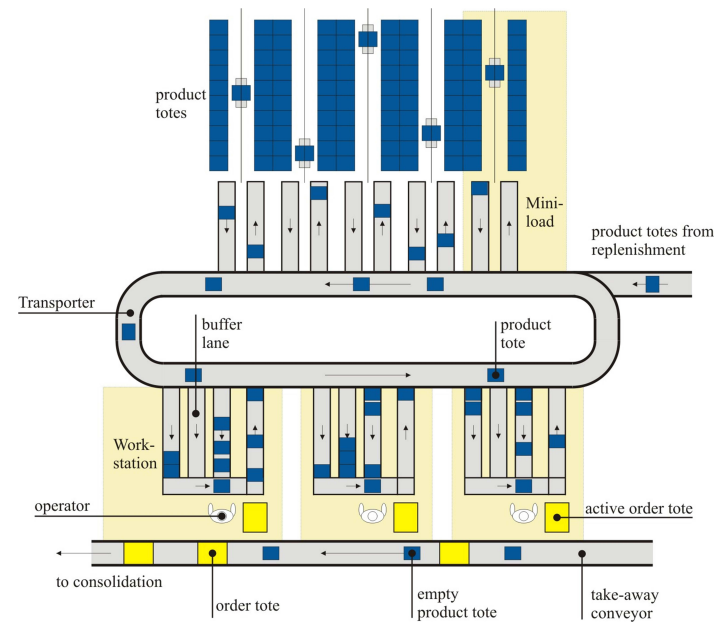

(a) System layout.

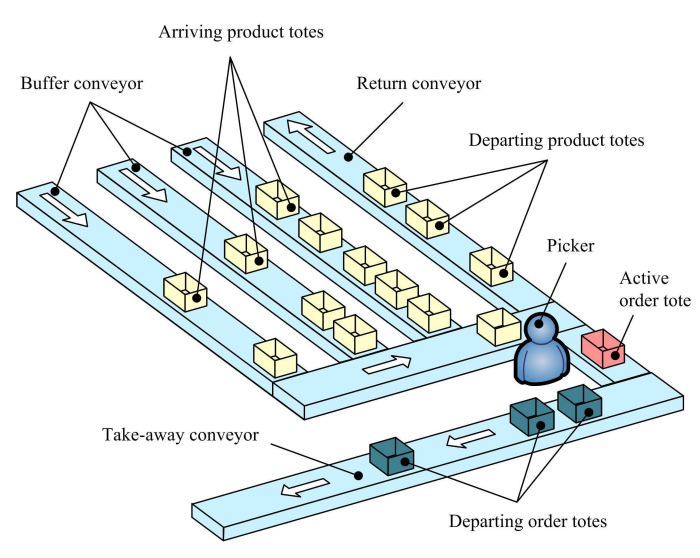

(b) Order picking workstation.

Figure 1: An automated warehouse with end-of-aisle order picking workstations.

Tote and order overtaking may occur at this workstation. A tote overtaking takes place when the next tote processed by the picker is not the oldest active tote in the buffer. We distinguish two types of tote overtaking. Overtaking by an available tote happens if the picker processes one of the active totes 
in the buffer, but not the oldest one. Overtaking by an unavailable tote happens when the picker does not pick any active tote, even though one or more active totes are present in the buffer. Instead, the picker waits for another active tote and picks this tote upon its arrival. Similarly, an order overtaking occurs when upon finishing an order the picker processes another order that is not the oldest in the buffer.

\section{AGGREGATE MODEL}

We propose an aggregate model as shown in Figure 2. It is essentially a single-server queueing system with an infinite buffer. Within the buffer there are a number of infinite queues, each containing totes for the same order. When the server is idle, an active tote in the buffer may be processed based on a so-called decision probability and overtaking distribution. The decision probability gives the probability that an active tote will be processed or not by the idle picker. The overtaking distribution is used to determine which active tote in the buffer will be selected as the next tote to be processed. The server then processes the selected active tote with a processing time sampled from the Effective Process Time (EPT) distribution.

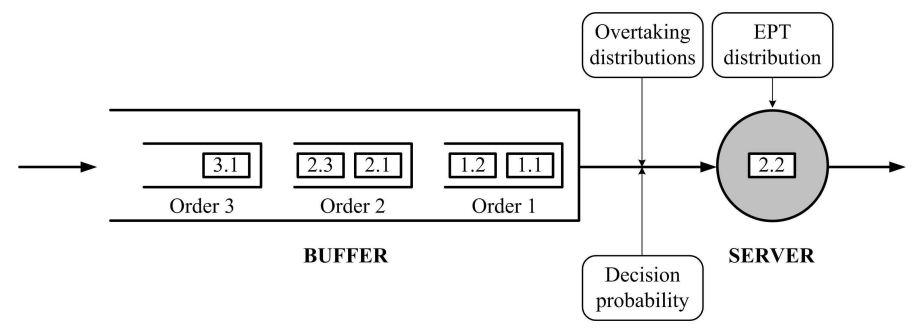

Figure 2: Aggregate model of an end-of-aisle order picking workstation with overtaking.

Figure 2 shows an example of both tote and order overtaking. The server is currently processing tote 2.2, which is the 2nd arriving tote of order 2. However, order 1 arrived earlier than order 2 (that is, order 1 is positioned in front of order 2). Hence, order 2 overtakes order 1 . Also, the 1st arriving tote of order 2 (tote 2.1) has not been processed yet. As such, the 2nd tote of order 2 overtakes the 1 st tote of order 2.

We measure the inputs for the aggregate model directly from tote arrival and departure data of an operating order picking workstation. Having the inputs, we then use the aggregate model to predict the mean and variability of tote and order flow times of the operating order picking workstation.

\subsection{Calculating EPTs}

We aggregate all process time components involved in an order picking workstation, e.g., raw pick time, setup time, and outages, picker unavailability, breakdowns, etc., into a single EPT distribution. The EPTs are obtained directly from arrival and departure data using either an EPT algorithm (e.g., Jacobs et al. (2003)) or using a sample path equation (e.g., Kock, Etman, and Rooda (2008)), depending on how the aggregate model is defined. For the aggregate model proposed here, we will use a sample path equation to calculate the EPTs.

To illustrate how EPTs are calculated, we use an example of arrivals $(A)$ and departures $(D)$ of three active totes as shown in Figure 3. There is no tote overtaking in this example; totes are processed in a FIFO sequence. The EPTs of the three totes are depicted at the bottom part of the figure. We use the following sample path equation to calculate the EPTs:

$$
\mathrm{EPT}_{i}=D_{i}-\max \left\{A_{i}, D_{i-1}\right\} .
$$

$D_{i}$ denotes the departure time of $i$-th departing tote. $A_{i}$ denotes the arrival time of the corresponding $i$-th departing tote. Note that the EPT of tote 1 (EPT 1) is comprised of a setup (time $0-3$ ) and picking (time 3-7), while the EPT of tote 3 (EPT 3) is comprised of picking (time 11-13.5 and time 15.5-18) and a disruption (time 13.5-15.5). 


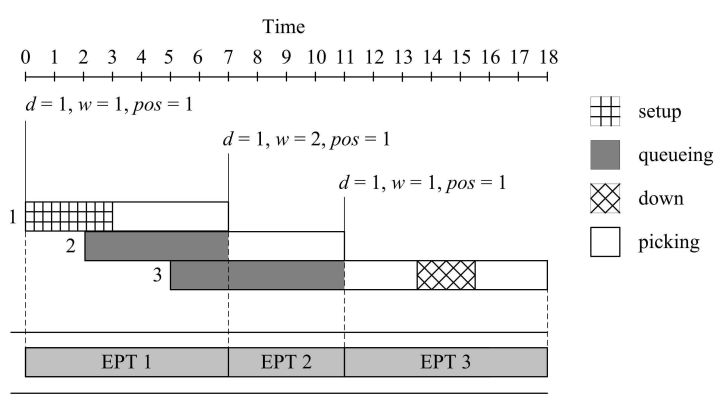

Figure 3: Tote-time diagram for active totes with order length $k=3$, FIFO processing.

An EPT distribution is created when the EPTs of all totes have been calculated. We use a gamma distribution to represent the EPT distribution, but other suitable distributions may be used as well. The EPT distribution is used to sample the processing times of totes in the aggregate model.

\subsection{Measuring Overtaking}

We model tote overtaking by means of wip-dependant overtaking distributions. The following variables are used:

$w=$ the number of active totes in the buffer.

$w_{\mathrm{R}}=$ the number of remaining active totes not yet arrived.

pos $=$ the position of the active tote in the buffer that is processed next by the picker.

Let us observe the tote-time diagram in Figure 3. The picker finishes processing tote 1 at time 7. At that moment, totes 2 and 3 are in the buffer and the next tote processed is the oldest tote (according to FIFO processing). Hence, at time 7 we have $w=2$ and pos $=1$. With the FIFO assumption, the value of pos will always be equal to 1 since the next tote to be processed is always the active tote located at the first position in the buffer.

Relaxing the FIFO assumption, we distinguish two types of tote overtaking, namely overtaking by an available tote and overtaking by an unavailable tote. Figure 4 depicts the two different types of tote overtaking.

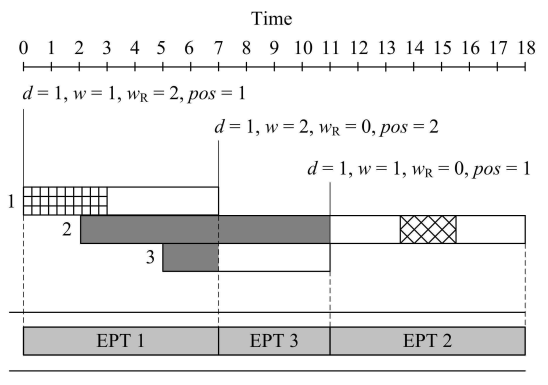

(a) Tote overtaking by an available tote.

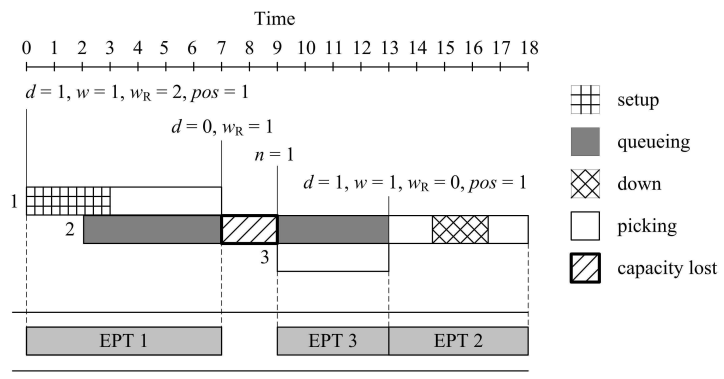

(b) Tote overtaking by an unavailable tote

Figure 4: Tote-time diagram for active totes with order length $k=3$, with tote overtaking.

Let $d$ be the decision of whether or not an active tote is immediately processed once the picker is idle and there is at least one active tote in the buffer, where:

$$
d= \begin{cases}1, & \text { A tote is processed. } \\ 0, & \text { A tote is not processed. }\end{cases}
$$


In the case of overtaking by an available tote (Figure 4(a)), a tote is always processed if the picker is idle and there is at least one active tote present in the buffer. Therefore, we always have $d=1$. The tote processed next is not the oldest active tote in the buffer. In Figure 4(a), tote 3 is processed earlier than tote 2 , although tote 2 arrived earlier than tote 3 . Hence, tote 3 overtakes tote 2 . At time 7 we register pos $=2$ since the active tote processed next (tote 3 ) is located at the second position in the buffer (behind tote 2). In general, pos $>1$ indicates a tote overtaking.

In the case of overtaking by an unavailable tote (Figure 4(b)), it is possible that a tote is not processed even though the picker is idle and there is an active tote present in the buffer. In Figure 4(b), the picker is idle at time 7 and tote 2 is present in the buffer. However, the picker does not immediately process this tote. Thus, we register $d=0$. Note that two out of three active totes have arrived (given order length $k=3$ ). Hence, we register the number of remaining active totes not yet arrived $w_{\mathrm{R}}=1$ at time 7 . Tote 3 arrives at time 9; it is processed immediately. At that moment we register the number of active totes that has been waited by the picker $n=1$, because there is only one new active tote waited for (tote 3 ) after $d=0$ at time 7. We consider time 7-9 as capacity loss.

Figure 5 illustrates order overtaking. There are three orders: $P, Q$, and $R$, which arrived at times 0,1 , and 8 , respectively. Note that the arrival time of an order is equal to the arrival time of the first tote of that order. Each order consists of two totes. Order $R$ overtakes order $Q$ at time 11. To measure order overtaking, we use the same variables $w$ and pos as in measuring tote overtaking. However, here $w$ represents the number of orders in the buffer and pos represents the position of the order that is processed next by the picker. These two variables are evaluated every time a picker has finished an order.

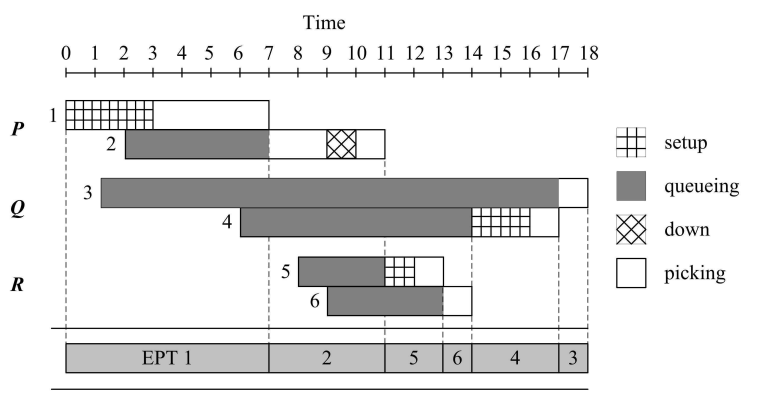

Figure 5: Tote-time diagram illustrating order overtaking.

A number of wip-dependant overtaking distributions and wip-dependant decision probabilities are created once all tote and order overtaking have been measured. For the overtaking distribution, the wip level represents the number of active totes (tote overtaking) or orders (order overtaking) in the buffer. For the decision probabilities, the wip level represents the number of remaining active totes not yet arrived.

\subsection{Predicting Flow Times}

We simulate the aggregate model with the EPT distribution, wip-dependant overtaking distributions, and wip-dependant decision probabilities as input. The mean and variability of tote and order flow times are then obtained.

The aggregate model works as follows. We generate totes in the aggregate model with an arrival process representing the operating order picking workstation. If the server is idle and the buffer contains one or more active totes, then we make a decision on whether or not one of the active totes is processed. This decision is based on the value sampled from the correct wip-dependant decision probabilities. Here, the wip corresponds to the number of remaining active totes not yet arrived. If it is decided that a tote should be processed, then we sample a wip-dependant tote overtaking distribution to determine which active tote will be processed. Here, the wip corresponds to the number of active totes in the buffer. On the contrary, if it is decided that none of the active totes in the buffer should be processed, then we sample the number of active totes that should be waited for. The server stays idle until the number of arriving active totes is equal to the sampled value. In this way, we model the capacity losses explicitly in the aggregate model. The selected tote will be sent to the server to be processed with a processing time sampled from the EPT distribution. If the picker has finished 
an order, the next order to be processed is determined using the wip-dependant order overtaking distribution.

The aggregate model gives tote and order flow times at output. Tote flow time is defined as the total time spent by a tote at the order picking workstation, which starts when a tote arrives at the workstation and ends when it departs the workstation. Order flow time is defined as the time required to complete an order, which starts when the first tote of an order arrives at the workstation and ends when the last tote of the order departs the workstation.

\section{MODEL VALIDATION}

We conducted a number of simulation experiments to validate the aggregate model. First, we need tote arrival and departure times to calculate the inputs for the aggregate model. Ideally, these arrival and departure times are obtained from a real, operating order picking workstation. However, for validation purposes we create a simulation model of the workstation in Figure 1(b) to generate tote arrival and departure times. These are generated at one utilization level called the training point. We refer to this "simulated" operating order picking workstation as the detailed model. Subsequently, we determine from the arrival and departure data the EPT distributions, overtaking distributions, and the decision probabilities. The detailed and aggregate models are then simulated at various utilization levels to compare the flow times from both models.

Some parameters used in the detailed model are as follows. Totes from three orders arrive simultaneously at the workstation according to a Poisson process with a total arrival rate of . Once all totes of an order have arrived, the totes from a new order start arriving. Hence, totes from three orders are continuously arriving. We assume a uniformly distributed order length in the range $\{1,2, \ldots, 20\}$. Four types of process time are modeled. Raw pick time is the time required to pick a tote. A setup, which includes activities such as moving the active order tote to the take away conveyor, scanning the barcode of a new order tote, and placing the new order tote at the pick position, is performed only when the picker processes the first tote of an order. Disruptions such as incorrect tote administration, unreadable barcode, or distraction from other pickers occur during order picking. Table 1 summarizes the values of parameters used in three experiments with the detailed model.

Table 1: Parameters for the detailed model.

\begin{tabular}{lcc}
\hline & Distribution type & Parameter(s) \\
\hline Raw pick time & Gamma & mean $=17.5$ seconds, SCV $=0.8$ \\
Setup time & Uniform & min $=10.0$ seconds, max $=15.0$ seconds \\
Time between disruptions & Exponential & mean $=30$ minutes \\
Disruptions length & Exponential & mean $=2$ minutes \\
\hline
\end{tabular}

In the first two experiments we create only tote overtaking in the detailed model. We assume that upon departure of a tote, all active totes in the buffer and a pre-defined number of remaining active totes not yet arrived have the same probability to be selected as the next tote to be processed. That is, the next tote to be processed is the $x$-th arriving active tote, which is selected with the probability

$$
p(x)=\frac{1}{N+\min \left\{n_{\mathrm{T}},(k-m)\right\}} .
$$

In this equation, $N$ is the number of active totes in the buffer, $k$ is the order length of the active order, $m$ is the number of active totes that have arrived at the buffer so far, and $n_{\mathrm{T}}$ is a user-defined tote overtaking parameter representing the number of remaining active totes not yet arrived that can also be selected as the next tote to be processed. Note that if all active totes have arrived $(m=k)$, the next tote to be processed will be one of the active totes in the buffer $(N)$. Figure 6 illustrates tote overtaking in the detailed model with $n_{\mathrm{T}}=3$ and order length $k=10$.

To model the two types of tote overtaking (see Section 3.2), we set $n_{\mathrm{T}}=\{0,1,3,5\}$. Overtaking by an available tote is modeled with $n_{\mathrm{T}}=0$, which suggests that the next tote to be processed may only be selected from the active totes present in the buffer. With $n_{\mathrm{T}}=\{1,3,5\}$, we model overtaking by an unavailable tote at different extents. 


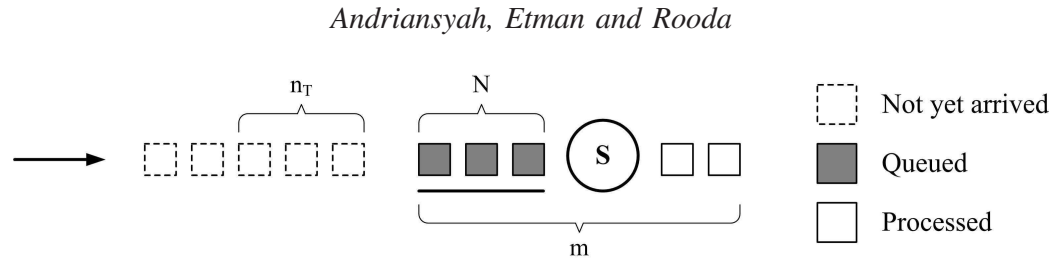

Figure 6: Tote overtaking in the detailed model: each active tote at positions $N+n_{\mathrm{T}}$ has the same probability given by Equation (2) to be selected as the next tote to be processed by the server $\mathrm{S}$.

In the third experiment we create both tote and order overtaking in the detailed model. For the tote overtaking we set $n_{\mathrm{T}}=\{0,3\}$. For the order overtaking we assume that when an order has been finished, each order in the buffer has the same probability to be selected as the next order.

The experimental setup is as follows. At each value of $n_{\mathrm{T}}$, arrival and departure data of 1,000,000 totes are generated from one simulation run of the detailed model. This is performed at training point $u=0.8$, where $u=/ \max$, is the total tote arrival rate, and $\max$ is the maximum throughput. To obtain the mean and variability of flow times, we run the detailed model and the aggregate model at utilization levels $u=\{0.30,0.32,0.34, \ldots, 0.98\}$. Fifty simulation runs are performed at each utilization level for both models. Each run length covers 300,000 totes excluding a warm up period of 30,000 totes.

Figure 7 depicts the frequency and probability of tote overtaking under different values of $n_{\mathrm{T}}$ from $1,000,000$ totes. The frequency of overtaking by available totes increases with $n_{\mathrm{T}}$, while the frequency of overtaking by unavailable totes decreases slightly with larger $n_{\mathrm{T}}$ (see Figure 7(a)). Yet, larger $n_{\mathrm{T}}$ leads to higher probability of overtaking many totes, as more remaining active totes that have not yet arrived may also be selected as the next tote to be processed (see Figure 7(b)).

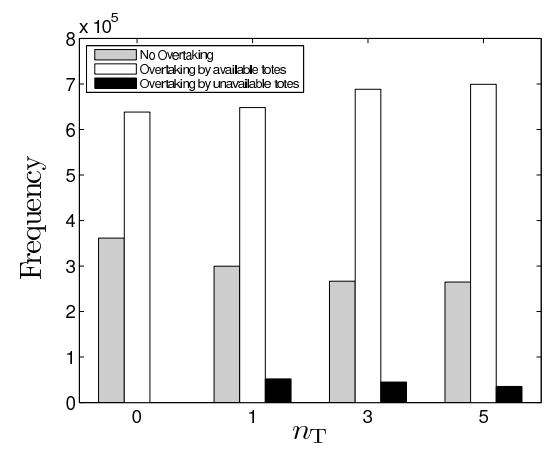

(a) Frequency.

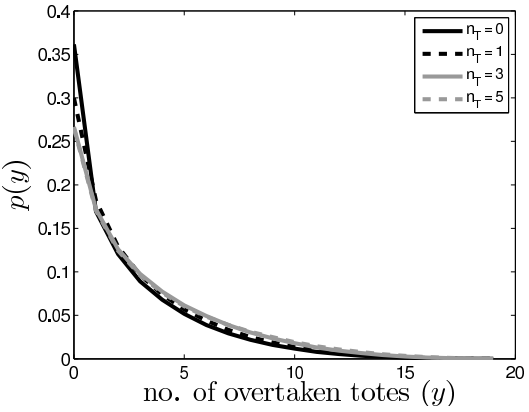

(b) Probability.

Figure 7: Tote overtaking under different values of $n_{\mathrm{T}}$.

\subsection{Tote Overtaking by an Available Tote}

For tote overtaking by an available tote $\left(n_{\mathrm{T}}=0\right)$, each active tote in the buffer has the same probability of $1 / N$ to be selected as the next tote (see Equation 2).

Figure 8 shows the EPT distributions and some of the wip-dependant overtaking distributions measured from tote arrival and departure data. There are two EPT distributions as shown in Figure 8(a), namely the EPTs of the first tote of an order (EPTs 1st) and the EPTs of the remaining totes of an order (EPTs 2+). We sort the EPTs into two distributions because these two sorts of EPTs are not identically distributed. This is because EPTs 1st always contain setup times, whereas no setup time is involved in EPTs 2+. This approach is referred to as the 1st tote difference EPT approach (Andriansyah, Etman, and Rooda 2009). The significant difference between EPTs 1st and EPTs 2+ can be seen from their CDF (Cumulative Distribution Function) in Figure 8(a).

Figure 8(b) depicts tote overtaking distributions for the number of active totes in the buffer $w=\{2,4,6,8,10\}$. Since each active tote has the probability of $1 / N$ to be selected as the next tote according to Equation (2), we expect to see a uniform distribution for all values of $w$. This is verified in 
Figure 8(b), where the active totes at the 1st until the $w$-th position in the buffer have equal probability to be selected.

In the case of tote overtaking by an available tote, an idle picker always processes an active tote as long as there is at least one active tote in the buffer (see Figure 4(a)). This is modeled explicitly in the aggregate model.

Figure 9 compares the mean flow times at various utilization levels $u$ from the detailed model and the aggregate model. The flow time prediction by the aggregate model is very accurate as compared to the detailed model, with prediction errors of less than $0.25 \%$ for the mean flow times and $5.3 \%$ for the Squared Coefficient of Variation (SCV) of the flow times at all simulated utilization levels.

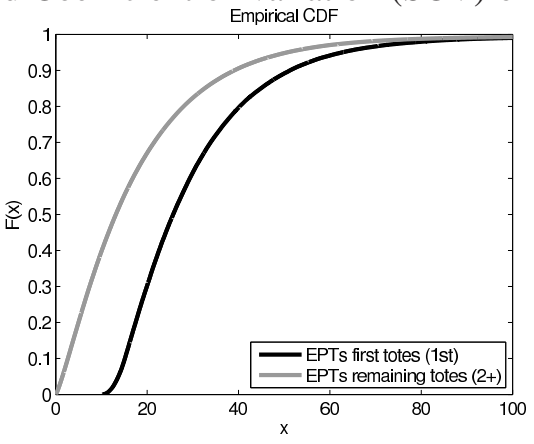

(a) $\mathrm{CDF}$ of EPTs.

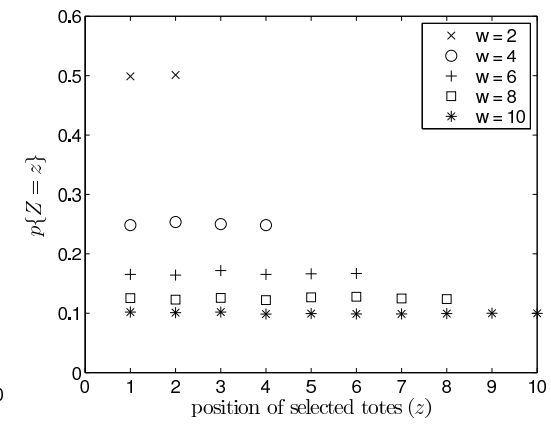

(b) Tote overtaking distributions for wip levels $w$.

Figure 8: Measured input for the aggregate model with tote overtaking by an available tote.

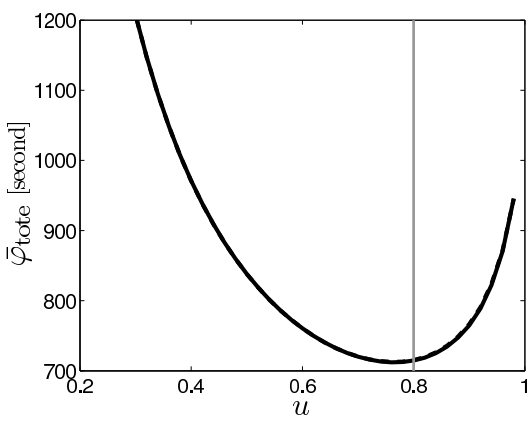

(a) Tote.

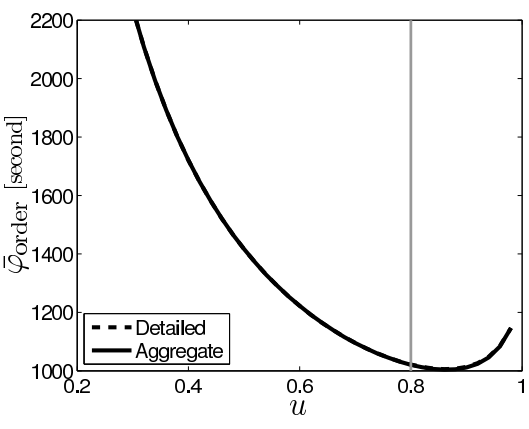

(b) Order.

Figure 9: Mean flow times in case of tote overtaking by an available tote.

\subsection{Tote Overtaking by an Unavailable Tote}

Tote overtaking by an unavailable tote is modeled in the detailed model by setting $n_{\mathrm{T}}=\{1,3,5\}$. By having different values of $n_{\mathrm{T}}$, we investigate the effect of intensity of overtaking by an unavailable tote on the flow time prediction accuracy by the aggregate model.

Figure 10 depicts the wip-dependant decision probabilities $p(1)$ of processing an active tote in the buffer when the picker is idle. In this figure, the wip level $w_{\mathrm{R}}$ indicates the number of remaining active totes that have not arrived. As expected, $p(1)$ decreases with increasing $w_{\mathrm{R}}$. That is, the more remaining active totes not yet arrived, the lower the probability that one of the active totes in the buffer is processed. We also verify that larger $n_{\mathrm{T}}$ leads to lower $p(1)$, given the same utilization level. This is because larger $n_{\mathrm{T}}$ allows for more remaining active totes not yet arrived to be selected, hence lower $p(1)$. Note that these decision probabilities are measured from the detailed model at utilization level $u=0.8$ and are used in the aggregate model to predict the flow times at various utilization levels $u=\{0.30,0.32,0.34, \ldots, 0.98\}$.

At all three settings, the aggregate model accurately predicts the mean and variability of tote and order flow times. The prediction errors for the mean tote and order flow times are less than $3.71 \%$ and $2.18 \%$, respectively. The prediction errors for the SCV of tote and order flow times are less than 


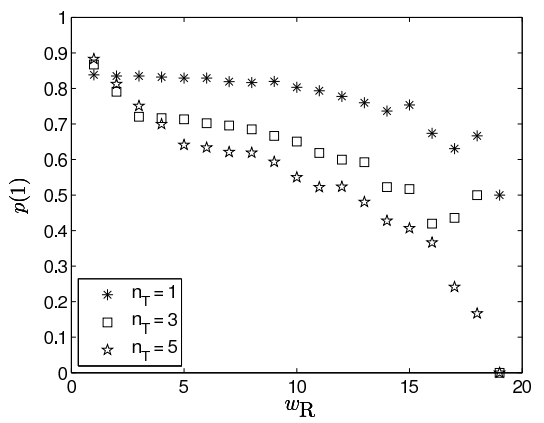

Figure 10: Measured decision probabilities from the detailed model at utilization level $u=0.8$.

$8.54 \%$ and $3.00 \%$, respectively. Figure 11 compares the mean tote and order flow times from the detailed and the aggregate model.

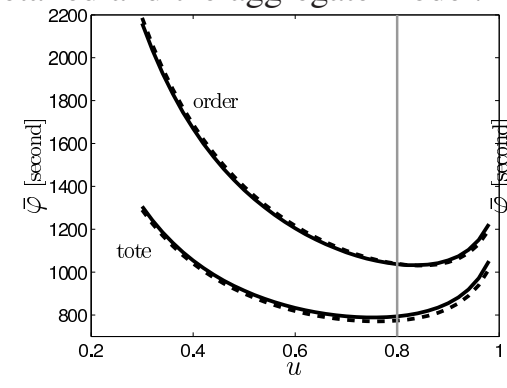

(a) $n_{\mathrm{T}}=1$

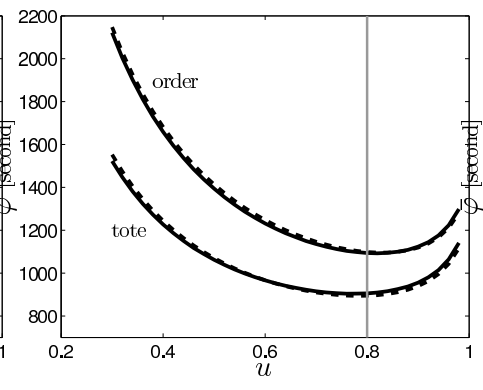

(b) $n_{\mathrm{T}}=3$.

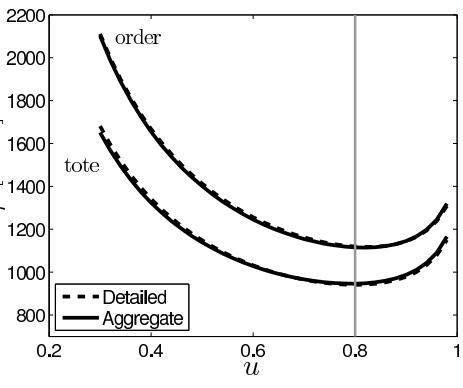

(c) $n_{\mathrm{T}}=5$.

Figure 11: Mean tote flow times in case of tote overtaking by an unavilable tote.

\subsection{Tote and Order Overtaking}

We now consider the case where tote and order overtaking happen at the workstation. We allow tote overtaking by an available and an unavailable tote. Order overtaking occurs only between available orders; each order in the buffer has an equal probability to be selected as the next order.

Figure 12 compares the results from the aggregate model with and without order overtaking distribution to the results from the detailed model with $n_{T}=3$. Without overtaking distribution, the aggregate model assumes a FIFO processing of orders. The figures suggest that the order overtaking distribution is significant to flow time prediction accuracy. This is because order overtaking happens very often in the detailed model. Furthermore, the flow time prediction by the aggregate model is less accurate at very high and very low utilization levels. Recall that the wip-dependant order overtaking distributions are obtained from the detailed model run at training point $u=0.8$ and then used for all utilization levels in the aggregate model. However, when the aggregate model is run at a high utilization level (e.g., 0.98) it is very likely that an order overtaking distribution required for a high wip level is not available, simply because this high wip level was not encountered at the training point. In this case, we use the order overtaking distribution for the maximum available wip level instead. This way of compromising for missing order overtaking distribution decreases the prediction accuracy. Note that the aggregate model predicts the flow times more accurately given a detailed model with $n_{T}=0$.

\section{CASE STUDY}

A real, operating automated warehouse is used as a case study to show the application of the proposed aggregate modeling methodology. We consider an automated warehouse that supplies a number of supermarket chains in the Netherlands. This warehouse consists of five miniloads, a conveyor loop, and three end-of-aisle order picking workstations as shown previously in Figure 1(a). 
Andriansyah, Etman and Rooda

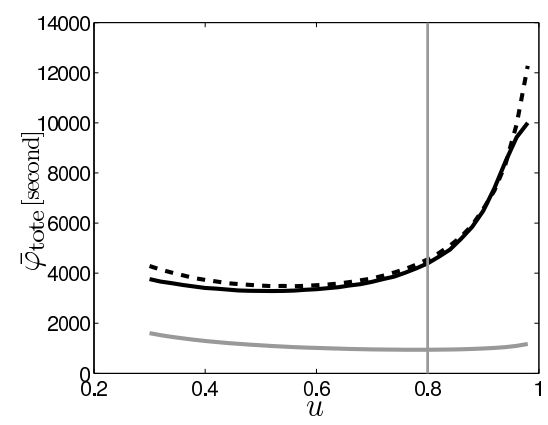

(a) Tote.

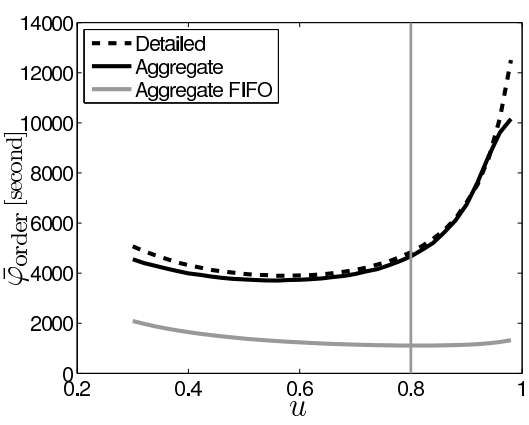

(b) Order.

Figure 12: Mean flow times in case of tote and order overtaking, $n_{T}=3$.

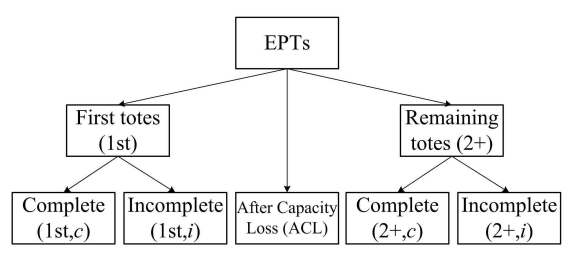

(a) Sorts.

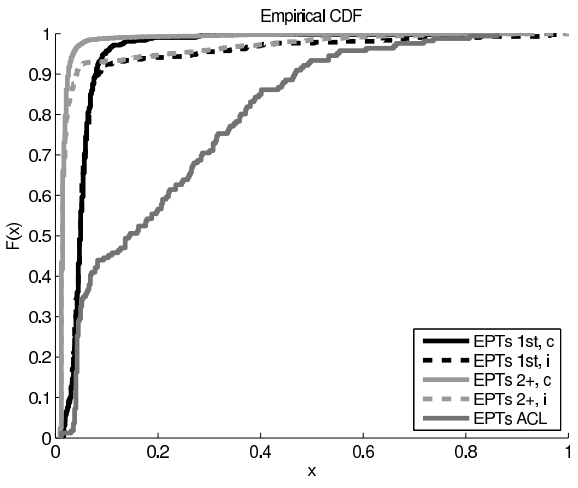

(b) CDF.

Figure 13: Different sorts of EPTs measured from the case study data.

We collected tote arrival and departure data of three working days from all three workstations. The data is comparable to the one that was generated using the detailed model for validation purposes in the previous section. Each tote arrival or departure contains information about the time of occurrence, tote ID, order ID, and order length. A total of $18.3 \%$ tote overtaking has been measured, in which $15.9 \%$ is overtaking by available totes and the remaining $2.4 \%$ is overtaking by unavailable totes. There is also $4.6 \%$ order overtaking in the data.

All parameters for the aggregate model are obtained only from tote arrival and departure data. We measure separately for each workstation the overtaking distributions, the decision probabilities, and the EPT distributions. Subsequently, we reconstruct the arrival process of totes at each workstation such that the interarrival time of totes and the interarrival time of orders are correctly represented.

A closer examination to the EPTs measured from the data reveals that there are several sorts of EPTs. As expected, the EPTs of the first totes of an order are larger than the EPTs of the remaining totes of an order due to the setup applied to every first totes of an order. Furthermore, we observed that many large EPTs occur when not all totes for the active order are present in the buffer. A possible cause is that the pickers hesitate to wait for totes so they leave the workstations when the totes are not yet complete in the buffer. Since time during which the picker leaves the workstation is included in the EPT of the next tote to be processed, a large EPT occurs. As such, we sort the EPTs based on the completeness of totes in the buffer when the picker starts picking a tote. We also consistently found in the data that EPTs of the first totes after a capacity loss (e.g., EPT of tote 3 in Figure 4(b)) are substantially larger than other EPTs. The reason is because these EPTs include the time when the pickers actually have not returned yet after leaving the workstation following a capacity loss. Thus, we also treat these EPTs separately and label them as EPT After Capacity Loss (ACL). Figure 13 shows the different sorts of EPT and the Cumulative Distribution Functions (CDF) of EPTs for workstation 1. 


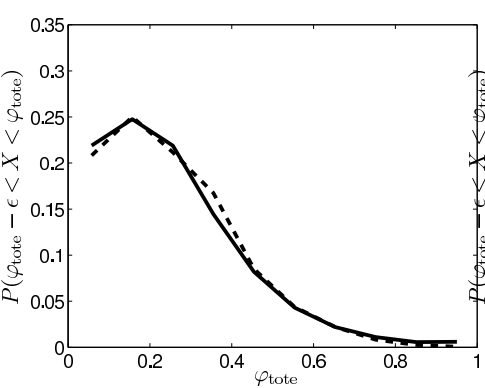

(a) Workstation 1 .

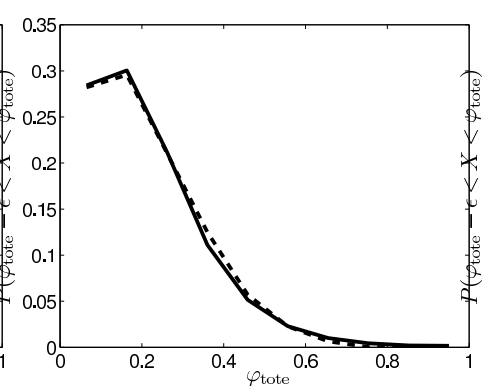

(b) Workstation 2 .

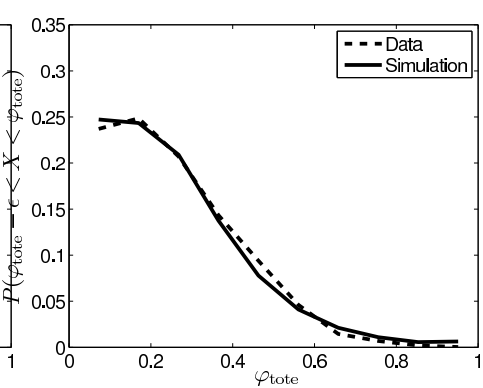

(c) Workstation 3 .

Figure 14: Tote flow time distributions.

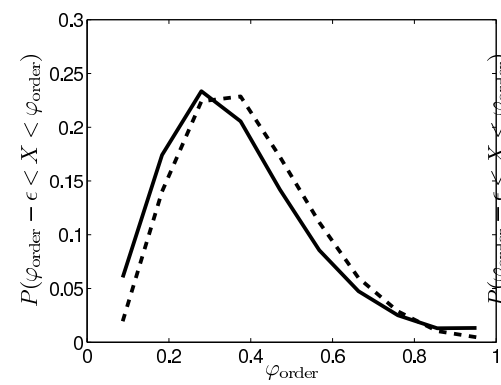

(a) Workstation 1 .

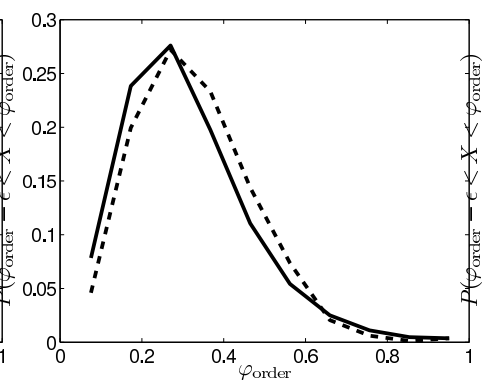

(b) Workstation 2 .

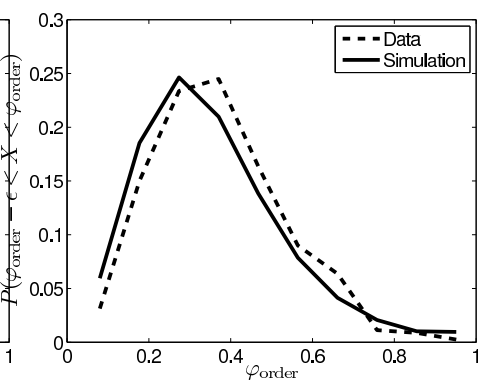

(c) Workstation 3 .

Figure 15: Order flow time distributions.

We simulated the aggregate model with 50 replications, each with a run length of 1,000,000 totes excluding a warm-up period of 300,000 totes. We simulate each workstation separately. The resulting flow time distributions from the aggregate model are compared with those measured from the data. Figures 14 and 15 show the comparisons for all three workstations. Note that in reality, the three workstations operate within limited working hours. For non steady-state analysis, we also performed 50 simulation runs in which the simulation is terminated once the number of product totes processed in one working day has been reached. The results are compared to the real data obtained from the three working days.

In general, the tote flow time distributions are accurately predicted by the aggregate model for all three workstations. The prediction accuracy of order flow time distributions is slightly less compared to that of tote flow time distributions. The prediction errors for the mean tote and order flow times are less than $1.35 \%$ and $6.31 \%$, respectively. The absolute difference of SCV of tote and order flow times between the data and the aggregate model are less than 0.11 and 0.08 , respectively. These values applies to all three workstations. Results from the non steady-state simulation also shows that the $95 \%$ confidence interval of the mean flow time from the aggregate model contains the flow times from the real data.

\section{CONCLUSIONS}

In this paper we have proposed an aggregate modeling methodology to predict the flow time performance of an end-of-aisle order picking workstation with product overtaking. We require only limited, measurable data namely the arrival and departure data of totes. Based on this data, we reconstruct the effective process time distribution, overtaking distribution, and the decision probability to be used in the aggregate model. Using a number of simulation experiments, we have shown that the aggregate model predicts flow time of product and order accurately. We have also applied the methodology to data obtained from a real, operating automated warehouse. The resulting flow time prediction shows satisfactory accuracy.

The proposed aggregate modeling methodology should be of value for analyzing system performance under different settings of order release strategies, product interarrival rates, order length 
distributions, etc. We also foresee the use of effective process time as a real-time performance monitoring tool for such an order picking workstation.

Future research will be directed towards aggregate modeling methodology for end-of-aisle order picking workstations where pickers are allowed to process multiple orders simultaneously. This particular type of system is often encountered in practice since it allows for significantly higher throughput.

\section{ACKNOWLEDGMENTS}

This work has been carried out as part of the FALCON project under the responsibility of the Embedded Systems Institute with Vanderlande Industries as the industrial partner. This project is partially supported by the Netherlands Ministry of Economic Affairs under the Embedded Systems Institute (BSIK03021) program. We would like to thank Ivo Adan, Jacques Resing, and Liqiang Liu of the Department of Mathematics and Computer Science at the Eindhoven University of Technology, and Roelof Hamberg from the Embedded Systems Institute for invaluable discussions during the model development. We also thank Bruno van Wijngaarden from Vanderlande Industries for providing the data used in the case study.

\section{REFERENCES}

Andriansyah, R., L. F. P. Etman, and J. E. Rooda. 2009. Simulation model of a single-server order picking workstation using aggregate process times. In Proceedings of the 1st International Conference on Advances in System Simulation (SIMUL).

Gu, J., M. Goetschalckx, and L. F. McGinnis. 2010. Research on warehouse design and performance evaluation: a comprehensive review. European Journal of Operational Research 203:539-549.

Hopp, J. W., and M. L. Spearman. 2008. Factory physics: Foundation of manufacturing management. 3 ed. London: McGraw Hill.

Jacobs, J. H., L. F. P. Etman, E. J. J. van Campen, and J. E. Rooda. 2003. Characterization of operational time variability using effective processing times. IEEE Transactions on Semiconductor Manufacturing 16 (3): 511-520.

Kock, A. A. A., L. F. P. Etman, and J. E. Rooda. 2008. Effective process times for multi-server flowlines with finite buffers. IIE Transactions 40 (3): 177-186.

Koo, P. H. 2009. The use of bucket brigades in zone order picking systems. OR Spectrum 31:759-774.

Rouwenhorst, B., B. Reuter, V. Stockrahm, G. J. van Houtum, R. J. Mantel, and W. H. M. Zijm. 2000. Warehouse design and control: framework and literature review. European Journal of Operational Research 122:515-533.

Yu, M., and R. de Koster. 2008. Performance approximation and design of pick-and-pass order picking systems. IIE Transactions 40:1054-1069.

Yu, M., and R. de Koster. 2009. The impact of order batching and picking area zoning on order picking system performance. European Journal of Operational Research 198:480-490.

\section{AUTHOR BIOGRAPHIES}

RICKY ANDRIANSYAH is a PhD student in the Systems Engineering group of the department of Mechanical Engineering at the Eindhoven University of Technology. His research work is on the development of aggregate modeling methodologies for performance quantification and analysis of automated warehouses. His email address is $<r$. andriansyah@tue.nl $>$.

PASCAL ETMAN is an associate professor in the Systems Engineering group of the department of Mechanical Engineering at the Eindhoven University of Technology. His research interests include simulation-based optimization, multidisciplinary design optimization, and the effective process time method for performance analysis of manufacturing systems. His email address is $<$ l.f.p.etman@tue.nl>.

JACOBUS ROODA is professor of the Systems Engineering group of the department of Mechanical Engineering at the Eindhoven University of Technology. His research interests include design and analysis of manufacturing systems, manufacturing control, and supervisory machine control. His email address is $<j \cdot e . r o o d a @ t u e . n l>$. 